\title{
Singularity Induced Interior Stokes Flows
}

\author{
N. Akhtar, G. A. H. Chowdhury \\ Department of Mathematics, Shahjalal University of Science and Technology, Sylhet, Bangladesh
}

Email address:

gahc_mat@yahoo.com (G. A. H. Chowdhury)

\section{To cite this article:}

N. Akhtar, G. A. H. Chowdhury. Singularity Induced Interior Stokes Flows. International Journal of Theoretical and Applied Mathematics. Vol. 3, No. 1, 2017, pp. 1-10. doi: 10.11648/j.jitam.20170301.11

Received: September 18, 2016; Accepted: November 10, 2016; Published: December 9, 2016

\begin{abstract}
Three complex variable circle theorems for studying the two-dimensional Stokes flows interior to a circular cylinder are presented. These theorems are formulated in terms of the complex velocities of the fundamental singularities in an unbounded incompressible viscous fluid. Illustrative examples are given to demonstrate their usefulness.
\end{abstract}

Keywords: Complex Variable Theory, Rotlet, Stokeslet, Stresslet, Stokes Flows

\section{Introduction}

The solutions of fluid mechanical problems involving fundamental singularities in the presence of rigid boundaries are of considerable interest in practice. Except in a few simple cases the solution of such a problem involving an arbitrary boundary is still, in general exceedingly difficult. In the case of two-dimensional slow flow theory there is a complex variable circle theorem [1] for the solutions of Stokes flows due to singularities outside a circular cylinder which corresponds to Milne-Thomson's circle theorem [2, 3] for potential flow outside the same cylinder, in the inviscid flow theory. Again it is notable that the same complex variable circle theorem can solve, in particular, some particular Stokes flow problems which cannot be done by the application of the real variable circle theorem [4, 5]. Moreover, the "condition for zero perturbation velocity" referred to in the former theorem may suggest, in many cases, relatively easily the strengths or positions or both, of the singularities of the basic flow so that the viscous flows outside the circular boundary exist. Again the literature for Stokes flows in the region interior to a circular cylinder is not as wide as that for the Stokes flows outside the same cylinder; and its mathematical treatment is mostly known in terms of the real variables, nearly polar co-ordinates $(r, \theta)$. Notably, Ranger [6] and Meleshko and Arof [7] have independently solved the problem for a single rotlet within a circular cylinder. Sen [5] has pointed out a method to solve some problems of slow viscous fluid flow within a circular cylinder with the aid of his circle theorems for the flows outside a circular boundary. Chowdhury and Sen $[8,9]$ have solved the problem of viscous fluid motion due to a Stokeslet within a circular cylinderical container. Daripa and Palaniappan [10] have extensively studied the singularity (rotlet or Stokeslet) driven Stokes flows interior and exterior to a circular cylinder. Here our object is to study Stokes flow interior to a circular cylinder in the light of the complex variable theory and to establish a number of complex variable circle theorems for slow viscous fluid motion within a circular cylinder in terms of the complex velocities of the fundamental singularities found in Chowdhury and Sen [11]; these theorems also correspond to the complex variable circle theorems for potential flow $[12,13]$ in an inviscid fluid within the same cylinder.

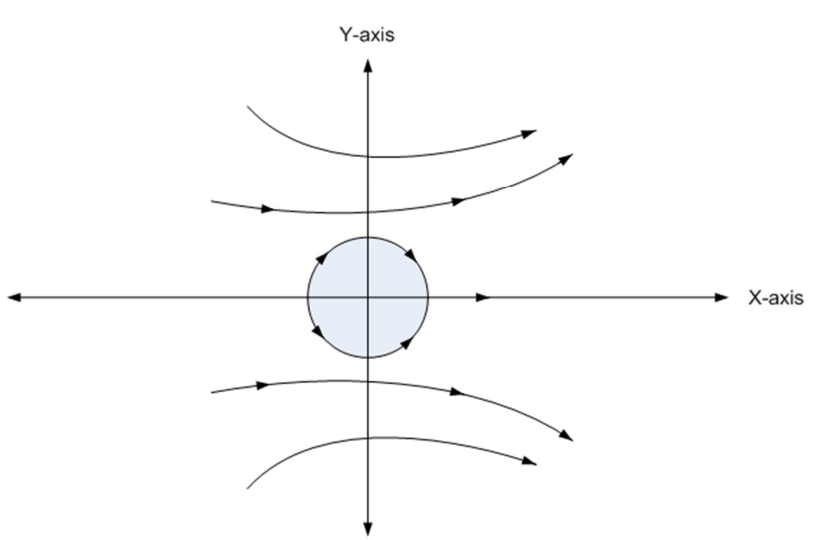

Figure 1. The streamlines for a line Strokeslet at the origin, which is directed along the positive $x$-axis. 


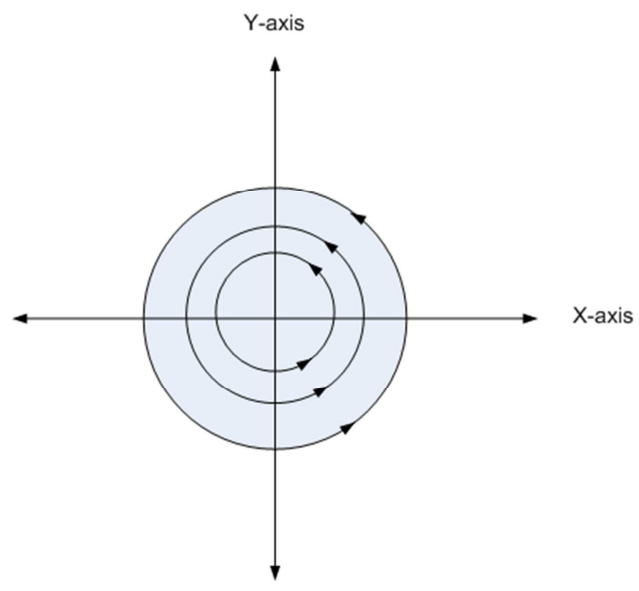

Figure 2. The streamlines for a line rotlet at the origin.

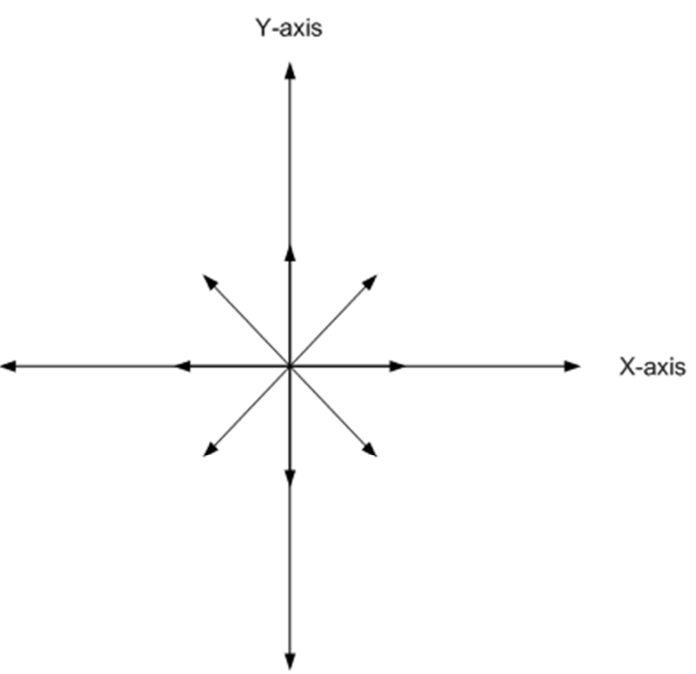

Figure 3. The streamlines for a stresslet at the origin.

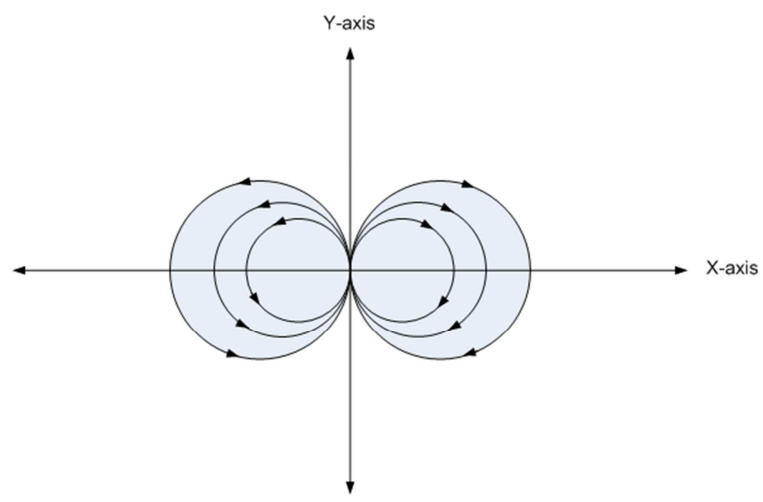

Figure 4. The streamlines for a potential doublet at the origin.

\section{The Interior Circle Theorems}

In this section we attempt to study in some detail the twodimensional slow viscous flows interior to a circular cylinder with the help of the complex analysis and we establish three complex variable circle theorems for the determination of such flows. This is done by making use of the fundamental solutions of the Stokes equations presented somewhat differently in Chowdhury and Sen [11], Milne-Thomson [3] and Langlois [14] and these solutions governing generally the two-dimensional Stokes flows are the complex velocity and the stream function which are respectively

$$
v(z, \bar{z})=\bar{W}(\bar{z})-\bar{z} \frac{d}{d z} W(z)-\omega(z),
$$

and

$$
\psi=\frac{1}{2} \mathrm{i}\left(\mathrm{z} \overline{\mathrm{W}}(\overline{\mathrm{z}})-\overline{\mathrm{z}} \mathrm{W}(\mathrm{z})+\int \bar{\omega}(\overline{\mathrm{z}}) \mathrm{d} \overline{\mathrm{z}}-\int \omega(\mathrm{z}) \mathrm{dz}\right),
$$

where $\mathrm{W}(\mathrm{z})$ and $\omega(\mathrm{z})$ are arbitrary functions of $\mathrm{z}$.

Theorem 1. Let there be an irrotational two-dimensional flow in a viscous fluid with no rigid boundaries. Let the flow be represented by the complex velocity $v_{0}(z, \bar{z})=-\omega_{0}(z)$, whose singularities are all in the region $|\mathrm{z}| \leq \mathrm{a}$ and let $\omega_{0}(\mathrm{z}) \sim \mathrm{o}\left(\frac{\mathrm{i} \lambda}{\mathrm{z}}\right)$ for large $|\mathrm{z}|, \mathrm{i} \lambda$ being a pure complex number. If a circular cylinder $|z|=a$ is now inserted into flow field, the complex velocity and the stream function for the flow inside the cylinder becomes respectively

$$
v(z, \bar{z})=-\omega_{0}(z)+\omega_{0}\left(\frac{a^{2}}{\bar{z}}\right)+\left(\frac{a^{2}}{z}-\bar{z}\right)\left(\frac{d}{d z} \bar{\omega}_{0}\left(\frac{a^{2}}{z}\right)+\frac{i \lambda}{a^{2}}\right)
$$

and

$$
\psi=\frac{1}{2} \mathrm{i}\left(\mathrm{z} \overline{\mathrm{W}}(\overline{\mathrm{z}})-\overline{\mathrm{zW}}(\mathrm{z})+\int \bar{\omega}(\overline{\mathrm{z}}) \mathrm{d} \overline{\mathrm{z}}-\int \omega(\mathrm{z}) \mathrm{dz}\right)
$$

where

$$
\mathrm{W}(\mathrm{z})=\bar{\omega}_{0}\left(\frac{\mathrm{a}^{2}}{\mathrm{z}}\right)+\frac{\mathrm{i} \lambda}{2 \mathrm{a}^{2}} \mathrm{z}
$$

and

$$
\omega(z)=\omega_{0}(z)-\frac{a^{2}}{z} \frac{d}{d z} \omega_{0}\left(\frac{a^{2}}{z}\right)-\frac{i \lambda}{z} .
$$

Proof. The proof consists in satisfying the following conditions.

(1) The complex function (3) is a complex velocity.

(2) The same function satisfies the no-slip conditions on $|\mathrm{z}|=\mathrm{a}$.

(3) The terms in (3) except, $-\omega_{0}(z)$, constitute the perturbation complex velocity, say

$$
v_{0}^{*}(\mathrm{z}, \overline{\mathrm{z}})=\omega_{0}\left(\frac{\mathrm{a}^{2}}{\overline{\mathrm{z}}}\right)+\left(\frac{\mathrm{a}^{2}}{\mathrm{z}}-\overline{\mathrm{z}}\right)\left(\frac{\mathrm{d}}{\mathrm{dz}} \bar{\omega}_{0}\left(\frac{\mathrm{a}^{2}}{\mathrm{z}}\right)+\frac{\mathrm{i} \lambda}{\mathrm{a}^{2}}\right) .
$$

It is to be shown that $v_{0}^{*}(z, \bar{z})$ introduces no singularities in the region $|z| \leq a$, and 
(4) $v_{0}^{*}(z, \bar{z})$, in particular, gives finite velocity at the origin.

Substituting the value of complex functions $\mathrm{W}(\mathrm{z})$ and $\mathrm{w}(\mathrm{z})$ from the formulas (5) and (6) respectively, into (1), then obtained the particular complex velocity (3) which is satisfy the condition (1). The circular boundary $|z|=a$, implies $\mathrm{z} \overline{\mathrm{Z}}=\mathrm{a}^{2}$ by which the complex velocity (3) clearly gives $v(z, \bar{z})=0$, on the boundary $|z|=a$; this result implies at once that the radial and the azimuthal velocity components vanish on the same boundary, and thus no-slip conditions are satisfied. Next by the condition $\mathrm{w}_{0}(\mathrm{z}) \sim \mathrm{o}\left(\frac{\mathrm{i} \lambda}{\mathrm{z}}\right)$ for large $|z|$, referred to in the theorem, we may infer that $w_{0}(z)$ has a Laurent's expansion of the form

$$
\mathrm{w}_{0}(\mathrm{z})=\mathrm{i} \lambda \frac{1}{\mathrm{z}}+\lambda_{1} \frac{1}{\mathrm{z}^{2}}+\lambda_{2} \frac{1}{\mathrm{z}^{3}}+\ldots .
$$

where $\lambda_{1}, \lambda_{2}$, etc. are complex constants.

By making use of the expansion (8) in (7), the perturbation complex velocity $v_{0}^{*}(z, \bar{z})$ within the region $|z| \leq a$ becomes

$$
v_{0}^{*}(\mathrm{z}, \overline{\mathrm{z}})=\frac{2 \bar{\alpha}_{1}}{\mathrm{a}^{2}}+\frac{1}{\mathrm{a}^{2}}\left(\mathrm{i} \lambda-\frac{2 \bar{\alpha}_{1}}{\mathrm{a}^{2}} \mathrm{z}+\ldots .\right) \overline{\mathrm{z}}+\frac{3 \bar{\alpha}_{2}}{\mathrm{a}^{4}} \mathrm{z}+\ldots
$$

which shows that $v_{0}^{*}(z, \bar{z})$ introduces no singularities in the region $|z| \leq a$, and obviously yields finite velocity at the origin; thus the last two conditions (3) and (4) are satisfied. Hence the proof of the theorem is complete.

Example. A rotlet within a circular cylinder

Let the basic flow be due to a rotlet of strength ik at the point $\mathrm{z}_{0}$ in an unlimited viscous fluid, where $\left|\mathrm{z}_{0}\right|<\mathrm{a}$. Then the complex velocity [11] of the flow in this case is given by

$$
v_{0}(\mathrm{z}, \overline{\mathrm{z}})=\frac{\mathrm{ik}}{\mathrm{z}-\mathrm{z}_{0}}
$$

which may be rewritten, by following Theorem 1 ,as

$$
\mathrm{w}_{0}(\mathrm{z})=-\frac{\mathrm{ik}}{\mathrm{z}-\mathrm{z}{ }_{0}} \text {. }
$$

Here one at once sees that $\omega_{0}(z) \sim o\left(\frac{i k}{z}\right)$ for large $|z|$. Therefore, the theorem applies here, when the cylinder $|z|=a$ is introduced into the flow field characterized by the complex velocity (10); and thus the complex velocity for the new flow interior to cylinder $|\mathrm{z}|=\mathrm{a}$, becomes

$$
v(z, \bar{z})=i k\left\{\frac{1}{z-z_{0}}+\frac{a^{2}}{z_{0}^{2}} \frac{1}{\bar{z}-\frac{a^{2}}{z_{0}}}+\frac{1}{z_{0}}+\left(\bar{z}-\frac{a^{2}}{z}\right)\left\{-\frac{a^{2}}{\bar{z}_{0}^{2}} \frac{1}{\left(z-\frac{a^{2}}{\bar{z}_{0}}\right)^{2}}+\frac{1}{a^{2}}\right)\right\},
$$

that is,

$$
v(z, \bar{z})=i k\left\{\frac{1}{z-z_{0}}-\frac{1}{z-\frac{a^{2}}{\bar{z}_{0}}}-\frac{a^{2}}{\bar{z}_{0}^{2}} \frac{\bar{z}}{\left(z-\frac{a^{2}}{\bar{z}_{0}}\right)^{2}}+\frac{a^{4}}{\bar{z}_{0}^{2}} \frac{1}{z\left(z-\frac{a^{2}}{\bar{z}_{0}}\right)^{2}}+\frac{\bar{z}}{a^{2}}-\frac{1}{z}+\frac{1}{z_{0}}\right\},
$$

which on the appropriate reduction leads to the following standard form.

$$
v(z, \bar{z})=i k\left\{\begin{array}{c}
\left.\frac{1}{z-z_{0}}-\frac{1}{z-\frac{a^{2}}{\bar{z}_{0}}}+\frac{a^{2}}{z_{0}^{2}} \frac{1}{\bar{z}-\frac{a^{2}}{z_{0}}}-\frac{a^{2}}{\bar{z}_{0}^{2}} \frac{\bar{z}-\frac{a^{2}}{z_{0}}}{\left(z-\frac{a^{2}}{\bar{z}_{0}}\right)^{2}}\right) \\
+\frac{a^{2}}{\bar{z}_{0}}\left(1-\frac{a^{2}}{z_{0} \bar{z}_{0}}\right)
\end{array}\right\}
$$

where the terms, excluding the first one, constitute the image system exterior to the cylinder $|z|=a$, which thus consists of (1) a rotlet of strength, -ik, (2) a stresslet of strength $\frac{-2 \mathrm{ika}^{2}}{\overline{\mathrm{z}}_{0}^{2}}$, and (3) a potential doublet of strength $\frac{\mathrm{ika}^{2}\left(1-\frac{\mathrm{a}^{2}}{\mathrm{z}_{0} \overline{\mathrm{z}}_{0}}\right)}{\overline{\mathrm{z}}_{0}}$, each being at the point $\mathrm{z}=\frac{\mathrm{a}^{2}}{\overline{\mathrm{z}}_{0}}$, with a shear flow $\frac{\mathrm{ik} \overline{\mathrm{z}}}{\mathrm{a}^{2}}$ and a uniform stream $\frac{\mathrm{ik}}{\mathrm{z}_{0}}$, outside the same cylinder. The stream function, $\psi$ for the flow structure within the boundary $|z|=a$ may be obtained by making use of the complex functions (5), (6) and (11) in the formula (4); but it is convenient to use the complex velocity (14) in the same formula (see the appendix) in order to visualize $\psi$ as the algebraic form of the stream functions of the individual singularities referred in (14). Thus expressing the stream function $\psi$ in terms of the polar co-ordinates $(r, \theta)$ one gets

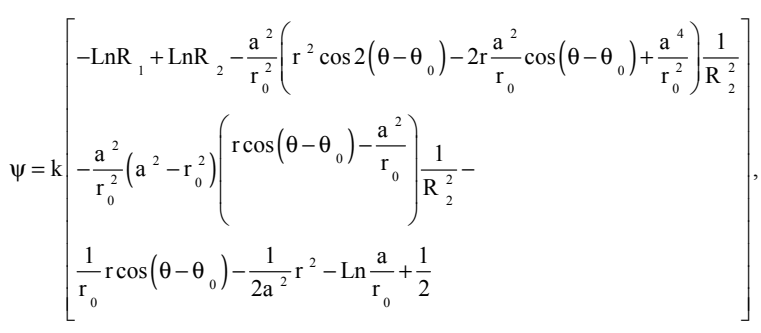

where 
$\mathrm{R}_{1}=\left|\mathrm{z}-\mathrm{z}_{0}\right|, \mathrm{R}_{2}=\left|\mathrm{z}-\frac{\mathrm{a}^{2}}{\overline{\mathrm{z}}_{0}}\right|, \mathrm{r}=|\mathrm{z}|, \mathrm{r}_{0}=\left|\mathrm{z}_{0}\right|, \theta=\arg \mathrm{z} \operatorname{and} \theta_{0}=\arg \mathrm{z}_{0}$.

The expression (15) may be made considerably simplified if one uses the result, $\mathrm{R}_{2}^{2}=\mathrm{r}^{2}+\frac{\mathrm{a}^{4}}{\mathrm{r}_{0}^{2}}-2 \mathrm{r} \frac{\mathrm{a}^{4}}{\mathrm{r}_{0}} \cos \left(\theta-\theta_{0}\right)$, in order to remove $\cos \left(\theta-\theta_{0}\right)$ from $\cos 2\left(\theta-\theta_{0}\right)$ shown in the same expression. Thus the stream function (15) simplifies to

$$
\psi=\mathrm{k}\left[-\mathrm{LnR}_{1}+\operatorname{LnR}_{2}+\left(\mathrm{a}^{2}-\mathrm{r}^{2}\right)\left\{\frac{1}{\mathrm{r}_{0}}\left(\mathrm{r} \cos \left(\theta-\theta_{0}\right)-\frac{\mathrm{a}^{2}}{\mathrm{r}_{0}}\right) \frac{1}{\mathrm{R}_{2}^{2}}+\frac{1}{2 \mathrm{a}^{2}}\right\}-\operatorname{Ln} \frac{\mathrm{a}}{\mathrm{r}_{0}}\right],
$$

from which Ranger's [6] stream function for the flow pattern due to a rotlet within circular cylinder is at once recovered by choosing $\mathrm{k}=-\mathrm{m}, \mathrm{a}=1, \mathrm{r}_{0}=\mathrm{c}, \theta_{0}=0$.

Theorem 2. Let $v_{0}(z, \bar{z})=\bar{\omega}_{0}(\bar{z})-\bar{z} \omega_{0}(z)-\omega_{0}(z)$ be the complex velocity for a motion in an unbounded viscous fluid, the singularities of $v_{0}(z, \bar{z})$ being all at a distance less than $a$ from the origin. Let $\mathrm{W}_{0}(\mathrm{z}) \sim \mathrm{o}(\alpha \operatorname{Lnz})$ and $\omega_{0}(z) \sim o\left(-\bar{\alpha} \operatorname{Ln} z+\frac{i \lambda}{z}\right)$ for large $|z|, \alpha$ being a complex constant, $\bar{\alpha}$ its complex conjugate and $i \lambda$ a pure complex constant. If a circular cylinder $|z|=a$ is now introduced into the flow field, the complex velocity $v(z, \bar{z})$ and the stream function $\psi$ for the flow inside the cylinder become

$$
v(z, \bar{z})=v_{0}(z, \bar{z})+v_{0}^{*}(z, \bar{z})
$$

and

$$
\psi=\frac{1}{2} \mathrm{i}\left(\mathrm{z} \overline{\mathrm{W}}(\overline{\mathrm{z}})-\overline{\mathrm{z}} \mathrm{W}(\mathrm{z})+\int \bar{\omega}(\overline{\mathrm{z}}) \mathrm{d} \overline{\mathrm{z}}-\int \omega(\mathrm{z}) \mathrm{dz}\right)
$$

where

$$
\begin{aligned}
& v_{0}^{*}(\mathrm{z}, \overline{\mathrm{z}})=-\frac{\bar{z}^{3}}{\mathrm{a}^{2}} \frac{\mathrm{d}}{\mathrm{d} \overline{\mathrm{z}}} \mathrm{W}_{0}\left(\frac{\mathrm{a}^{2}}{\overline{\mathrm{z}}}\right)-\overline{\mathrm{W}}_{0}\left(\frac{\mathrm{a}^{2}}{\mathrm{z}}\right)+\omega_{0}\left(\frac{\mathrm{a}^{2}}{\bar{z}}\right) \\
& -\bar{\alpha} \operatorname{Ln}\left(\frac{\mathrm{z} \overline{\mathrm{z}}}{\mathrm{a}^{2}}\right)+\left(\overline{\mathrm{z}}-\frac{\mathrm{a}^{2}}{\mathrm{z}}\right)\left\{\frac{\mathrm{d}}{\mathrm{dz}}\left(\frac{\mathrm{z}^{3}}{\mathrm{a}^{2}} \frac{\mathrm{d}}{\mathrm{dz}} \overline{\mathrm{W}}_{0}\left(\frac{\mathrm{a}^{2}}{\mathrm{z}}\right)-\bar{\omega}_{0}\left(\frac{\mathrm{a}^{2}}{\mathrm{z}}\right)\right)-\frac{\mathrm{i} \lambda}{\mathrm{a}^{2}}+\frac{\alpha}{\mathrm{z}}\right\},
\end{aligned}
$$

where

$$
\mathrm{W}(\mathrm{z})=\mathrm{W}_{0}(\mathrm{z})-\frac{\mathrm{z}^{3}}{\mathrm{a}^{2}} \frac{\mathrm{d}}{\mathrm{dz}} \overline{\mathrm{W}}_{0}\left(\frac{\mathrm{a}^{2}}{\mathrm{z}}\right)+\bar{\omega}_{0}\left(\frac{\mathrm{a}^{2}}{\mathrm{z}}\right)+\frac{\mathrm{i} \lambda}{2 \mathrm{a}^{2}} \mathrm{z}-\alpha \operatorname{Ln} \mathrm{z},
$$

and

$$
\begin{aligned}
& \omega(\mathrm{z})=\omega_{0}(\mathrm{z})+\overline{\mathrm{W}}_{0}\left(\frac{\mathrm{a}^{2}}{\mathrm{z}}\right)+\frac{1}{\mathrm{z}} \frac{\mathrm{d}}{\mathrm{dz}}\left(\mathrm{z}^{3} \frac{\mathrm{d}}{\mathrm{dz}} \overline{\mathrm{W}}_{0}\left(\frac{\mathrm{a}^{2}}{\mathrm{z}}\right)\right)-\frac{\mathrm{a}^{2}}{\mathrm{z}} \frac{\mathrm{d}}{\mathrm{dz}} \bar{\omega}_{0}\left(\frac{\mathrm{a}^{2}}{\mathrm{z}}\right)-\frac{\mathrm{i} \lambda}{\mathrm{z}} \\
& +\bar{\alpha} \operatorname{Ln} \mathrm{z}-\bar{\alpha} \operatorname{Ln}^{2}+\mathrm{a}^{2} \alpha \frac{1}{\mathrm{z}^{2}} .
\end{aligned}
$$

Proof. The proof of the theorem is complete if the following four conditions are satisfied.

(1) The expression (17) is a complex velocity.
(2) The same expression satisfies the no-slip conditions on the boundary $|z|=a$.

(3) $v_{0}^{*}(z, \bar{z})$ introduces no singularities in the region, $|\mathrm{z}| \leq \mathrm{a}$. and

(4) $v_{0}^{*}(z, \bar{z})$, in particular, gives finite velocity at the origin. By making use of the complex functions (20) and (21) in the general complex velocity

(1), we obtain the following particular complex velocity.

$$
v(\mathrm{z}, \overline{\mathrm{z}})=\overline{\mathrm{W}}_{0}(\overline{\mathrm{z}})-\overline{\mathrm{zW}}_{0}^{\prime}(\mathrm{z})-\omega_{0}(\mathrm{z})+v_{0}^{*}(\mathrm{z}, \overline{\mathrm{z}}),
$$

where

$$
\begin{aligned}
& v_{0}^{*}(\mathrm{z}, \overline{\mathrm{z}})=-\frac{\bar{z}^{3}}{\mathrm{a}^{2}} \frac{\mathrm{d}}{\mathrm{d} \overline{\mathrm{z}}} \mathrm{W}_{0}\left(\frac{\mathrm{a}^{2}}{\overline{\mathrm{z}}}\right)-\overline{\mathrm{W}}_{0}\left(\frac{\mathrm{a}^{2}}{\mathrm{z}}\right)+\omega_{0}\left(\frac{\mathrm{a}^{2}}{\overline{\mathrm{z}}}\right) \\
& -\bar{\alpha} \operatorname{Ln}\left(\frac{\mathrm{z} \overline{\mathrm{z}}}{\mathrm{a}^{2}}\right)+\left(\overline{\mathrm{z}}-\frac{\mathrm{a}^{2}}{\mathrm{z}}\right)\left\{\frac{\mathrm{d}}{\mathrm{dz}}\left(\frac{\mathrm{z}^{3}}{\mathrm{a}^{2}} \frac{\mathrm{d}}{\mathrm{dz}} \overline{\mathrm{W}}_{0}\left(\frac{\mathrm{a}^{2}}{\mathrm{z}}\right)-\bar{\omega}_{0}\left(\frac{\mathrm{a}^{2}}{\mathrm{z}}\right)\right)-\frac{\mathrm{i} \lambda}{\mathrm{a}^{2}}+\frac{\alpha}{\mathrm{z}}\right\} .
\end{aligned}
$$

Thus the condition (1) is satisfied. On the boundary $|z|=a$, we have $z \bar{z}=a^{2}$ so that the complex velocity (22) at once yields $v(z, \bar{z})=0$ on the same boundary; this satisfies the condition (2). Since by hypothesis, the singularities of $v_{0}(z, \bar{z})=\bar{W}_{0}(\bar{z})-\bar{z} W_{0}^{\prime}(z)-\omega_{0}(z)$, i.e., of $\mathrm{W}_{0}(\mathrm{z})$ and $\omega_{0}$ are all in the region $|z| \leq \mathrm{a}$. And since as referred to in the theorem, $\mathrm{W}_{0}(\mathrm{z}) \sim \mathrm{o}(\alpha \operatorname{Ln} \mathrm{z})$ and $\omega_{0}(\mathrm{z}) \sim \mathrm{o}\left(\bar{\alpha} \operatorname{Ln} \mathrm{z}+\frac{\mathrm{i} \lambda}{\mathrm{z}}\right)$ for large $|\mathrm{z}|$, both $\mathrm{W}_{0}(\mathrm{z})$ and $\omega_{0}(z)$ must have Laurent's expansions of the forms

$$
\mathrm{W}_{0}(\mathrm{z})=\alpha \operatorname{Ln} \mathrm{z}+\mathrm{a}_{1} \frac{1}{\mathrm{z}}+\mathrm{a}_{2} \frac{1}{\mathrm{z}^{2}}+\ldots \ldots . .
$$

and

$$
\omega_{0}(\mathrm{z})=-\bar{\alpha} \operatorname{Lnz}+\frac{i \lambda}{\mathrm{z}}+\mathrm{b}_{2} \frac{1}{\mathrm{z}^{2}}+\ldots \ldots . .
$$

where $\mathrm{a}_{1}, \mathrm{a}_{2}, \mathrm{~b}_{2}$ are complex constants. Substituting the complex functions(24) and (25) in (23) yields the perturbation complex velocity in the region $|z| \leq a$ as

$$
\begin{aligned}
v_{0}^{*}(\mathrm{z}, \overline{\mathrm{z}})= & 2\left(\bar{\alpha}+\frac{\overline{\mathrm{b}}_{2}}{\mathrm{a}^{2}}-\bar{\alpha} \operatorname{Lna}\right)-\left(\frac{3}{\mathrm{a}^{2}}\left(\overline{\mathrm{a}}_{1}-\frac{\overline{\mathrm{b}}_{3}}{\mathrm{a}^{2}}\right)+\frac{\overline{\mathrm{a}}_{1}}{\mathrm{a}^{2}}\right) \overline{\mathrm{z}} \\
& -\frac{4}{\mathrm{a}^{4}}\left(\overline{\mathrm{a}}_{2}-\frac{\overline{\mathrm{b}}_{4}}{\mathrm{a}^{2}}+\frac{\overline{\mathrm{a}}_{2}}{\mathrm{a}^{4}}\right) \mathrm{z}^{2}-\ldots \ldots \ldots \ldots+\frac{\mathrm{i} \lambda}{\mathrm{a}^{2}} \overline{\mathrm{z}}+\left(\frac{\mathrm{b}}{\mathrm{a}^{4}}+\frac{1}{\mathrm{a}^{2}}\right) \overline{\mathrm{z}}^{2} \\
& +\ldots \ldots-2\left(\bar{\alpha}+\frac{\overline{\mathrm{b}}_{2}}{\mathrm{a}^{2}}\right) \frac{1}{\mathrm{a}^{2}} \mathrm{z} \overline{\mathrm{z}}+\frac{3}{\mathrm{a}^{4}}\left(\overline{\mathrm{a}}_{1}-\frac{\overline{\mathrm{b}}_{3}}{\mathrm{a}^{2}}\right) \mathrm{z}^{2} \overline{\mathrm{z}}+\ldots \ldots \ldots \ldots
\end{aligned}
$$

which implies that $v_{0}^{*}(z, \bar{z})$ has no singularities within the space $|z| \leq a$ and, in particular, gives finite velocity at the origin; this satisfies the above two conditions (3) and (4). Thus the proof of the theorem is complete.

Example. A Stokeslet inside a circular cylinder 
Let the primary flow field in a viscous fluid be due to a Stokeslet of strength $\beta$ at the point $z_{0}$, where $\left|z_{0}\right|<a$; and the corresponding complex velocity [11] is given by

$$
\begin{aligned}
v_{0}(z, \bar{z}) & =-\frac{1}{2} \bar{\beta} \operatorname{Ln}\left(z-z_{0}\right)\left(\bar{z}-\bar{z}_{0}\right) \\
& +\frac{1}{2}\left\{\beta\left(\bar{z}-\bar{z}_{0}\right)+\bar{\beta}\left(z-z_{0}\right)\right\} \frac{1}{z-z_{0}},
\end{aligned}
$$

which may be expressed in the form

$$
v_{0}(\mathrm{z}, \overline{\mathrm{z}})=\overline{\mathrm{W}}_{0}(\overline{\mathrm{z}})-\overline{\mathrm{zW}} \mathrm{W}_{0}^{\prime}(\mathrm{z})-\omega_{0}(\mathrm{z})
$$

where

$$
\mathrm{W}_{0}(\mathrm{z})=-\frac{1}{2} \beta \operatorname{Ln}\left(\mathrm{z}-\mathrm{z}_{0}\right)
$$

and

$$
\omega_{0}(\mathrm{z})=\frac{1}{2} \bar{\beta} \operatorname{Ln}\left(\mathrm{z}-\mathrm{z}_{0}\right)+\frac{1}{2} \beta \overline{\mathrm{z}}_{0} \frac{1}{\mathrm{z}-\mathrm{z}_{0}}-\frac{1}{2} \bar{\beta} .
$$

We then easily calculate that the complex functions (29) and (30) are respectively $\mathrm{W}_{0}(\mathrm{z}) \sim \mathrm{o}(\alpha \operatorname{Ln} \mathrm{z})$ and $\omega_{0}(\mathrm{z}) \sim \mathrm{o}\left(\bar{\alpha} \operatorname{Ln} \mathrm{z}+\frac{\mathrm{i} \lambda}{\mathrm{z}}\right)$ for large $|\mathrm{z}|$, where $\quad \alpha=-\frac{1}{2} \beta$ and $i \lambda=\frac{1}{2}\left(\beta \bar{z}_{0}-\bar{\beta}_{0} z\right)$. This clearly shows that Theorem 2 applies here; and thus we may introduce the cylinder $|z|=a$ into the basic flow characterized by (27) and the complex velocity for the new flow inside the cylinder, after a somewhat lengthy calculation, emerges as

$$
\begin{aligned}
& v(z, \bar{z})=v_{0}(z, \bar{z})+\frac{1}{2} \frac{\beta}{z_{0}}\left(\bar{z}+\frac{a^{2}}{z_{0}}+\frac{a^{4}}{z_{0}^{2}} \frac{1}{\bar{z}-\frac{a^{2}}{z_{0}}}\right)+\frac{1}{2} \bar{\beta} \operatorname{Ln}\left(z-\frac{a^{2}}{\bar{z}_{0}}\right)+\frac{1}{2} \bar{\beta} \operatorname{Ln}\left(-\bar{z}_{0}\right) \\
& +\frac{1}{2} \bar{\beta} \operatorname{Ln}\left(\bar{z}-\frac{a^{2}}{z_{0}}\right)-\frac{1}{2} \beta \frac{\bar{z}_{0} a^{2}}{z_{0}^{2}} \frac{1}{\bar{z}-\frac{a^{2}}{z_{0}}}-\frac{1}{2} \beta \frac{\bar{z}_{0}}{z_{0}}-\frac{1}{2} \bar{\beta}+\frac{1}{2} \bar{\beta} \operatorname{Ln}\left(-z_{0}\right)-\frac{1}{2} \bar{\beta} \operatorname{Ln} a^{2} \\
& +\bar{z}\left(-\frac{1}{2} \bar{\beta} \frac{1}{\bar{z}_{0}}+\frac{1}{2} \bar{\beta}{\frac{a^{4}}{\bar{z}_{0}^{3}}}_{\left(z-\frac{a^{2}}{\bar{z}_{0}}\right)^{2}}-\frac{1}{2} \beta \frac{1}{z-\frac{a^{2}}{\bar{z}_{0}}}-\frac{1}{2} \bar{\beta} \frac{z_{0} a^{2}}{\bar{z}_{0}^{2}} \frac{1}{\left(z-\frac{a^{2}}{\bar{z}_{0}}\right)^{2}}+\frac{1}{2} \beta \frac{1}{z}\right)+ \\
& \frac{\bar{\beta}}{2}\left(\frac{a^{2}}{\bar{z}_{0}} \frac{1}{z-\frac{a^{2}}{\bar{z}_{0_{0}}}}-\frac{a^{4}}{\bar{z}_{0}^{2}} \frac{1}{\left(z-\frac{a^{2}}{\bar{z}_{0}}\right)^{2}}\right)+\frac{1}{2} \beta\left(-\bar{z}_{0} \frac{1}{z}+\bar{z}_{0} \frac{1}{z-\frac{a^{2}}{\bar{z}_{0}}}\right)+ \\
& \frac{1}{2} \bar{\beta}\left(z_{0} \frac{1}{z}-z_{0} \frac{1}{z-\frac{a^{2}}{\bar{z}_{0}}}+\frac{z_{0} a^{2}}{\bar{z}_{0}} \frac{1}{\left(z-\frac{a^{2}}{\bar{z}_{0}}\right)^{2}}\right)- \\
& \frac{1}{2} \beta \frac{a^{2}}{z^{2}}-\bar{z}\left(\frac{1}{2 a^{2}}\left(\beta \bar{z} \bar{z}_{0}-\bar{\beta} z_{0}\right)+\frac{1}{2} \beta \frac{1}{z}\right)+\frac{1}{2}\left(\beta \bar{z}_{0}-\bar{\beta} \quad z_{0}\right) \frac{1}{z}+\frac{1}{2} \beta \frac{a^{2}}{z^{2}} .
\end{aligned}
$$

The expression (31), after an appropriate simplification, takes the standard form

$$
\begin{aligned}
& v(z, \bar{z})=v_{0}(z, \bar{z})+\left\{\frac{1}{2} \bar{\beta} \operatorname{Ln}\left(\bar{z}-\frac{a^{2}}{z_{0}}\right)\left(z-\frac{a^{2}}{\bar{z}_{0}}\right)-\frac{1}{2}\left(\beta\left(\bar{z}-\frac{a^{2}}{z_{0}}\right)+\bar{\beta}\left(z-\frac{a^{2}}{\bar{z}_{0}}\right)\right) \frac{1}{\left(z-\frac{a^{2}}{\bar{z}_{0}}\right)}\right\}+
\end{aligned}
$$

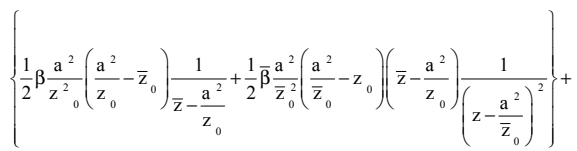

$$
\begin{aligned}
& \frac{1}{2} \bar{\beta} \frac{a^{2}}{\bar{z}_{0}^{3} z_{0}}\left(a^{2}-z_{0} \bar{z}_{0}\right)^{2} \frac{1}{\left(z-\frac{a^{2}}{\bar{z}_{0}}\right)^{2}}+\frac{1}{2}\left(\beta \bar{z} 0-\bar{\beta} z_{0}\right)\left(1-\frac{a^{2}}{z_{0} \bar{z}_{0}}\right) \frac{1}{z-\frac{a^{2}}{\bar{z}_{0}}}+ \\
& \frac{1}{2}\left(\beta \bar{z}_{0}-\bar{\beta}_{0}\right)\left(\frac{1}{z_{0} \bar{z}_{0}}-\frac{1}{a^{2}}\right) \bar{z}+\frac{1}{2} \beta\left(\frac{a^{2}}{z_{0}^{2}}-\frac{\bar{z}_{0}}{z_{0}}\right)+\frac{1}{2} \bar{\beta} \operatorname{Ln}\left(\frac{z_{0} \bar{z}_{0}}{a^{2}}\right),
\end{aligned}
$$

where the terms, excepting $v_{0}(z, \bar{z})$, constitute the image system in the region outside the boundary $|z|=a$, which thus consists of

(1) a Stokeslet of strength, $-\beta$,

(2) a stresslet of strength $\frac{1}{2} \bar{\beta} \frac{a^{2}}{\bar{z}_{0}^{2}}\left(\frac{a^{2}}{\bar{z}_{0}}-z_{0}\right)$,

(3) a potential doublet of strength $\frac{1}{2} \bar{\beta} \frac{a^{2}}{\bar{z}_{0}^{3} z_{0}}\left(a^{2}-z_{0} \bar{z}_{0}\right)^{2}$,

(4) a rotlet of strength $\frac{1}{2}\left(\beta \bar{z}_{0}-\bar{\beta} z_{0}\right)\left(1-\frac{a^{2}}{z_{0} \bar{z}_{0}}\right)$, each being at the point $\mathrm{z}=\frac{\mathrm{a}^{2}}{\overline{\mathrm{z}}_{0}}$,

(5) a shear flow and

(6) a uniform stream.

The evaluation of the corresponding stream function, $\psi$ for the flow pattern is somewhat tedious by making use of the complex functions (20), (21), (29) and (30) in the formula (18); but it is advantageous to use the complex velocity (32) in the same formula, in order to obtain directly the stream functions of the individual singularities (see the appendix). Finally, the form of the stream function $\psi$ in terms of polar coordinates $(r, \theta)$ characterizing the flow field (32), can be shown as

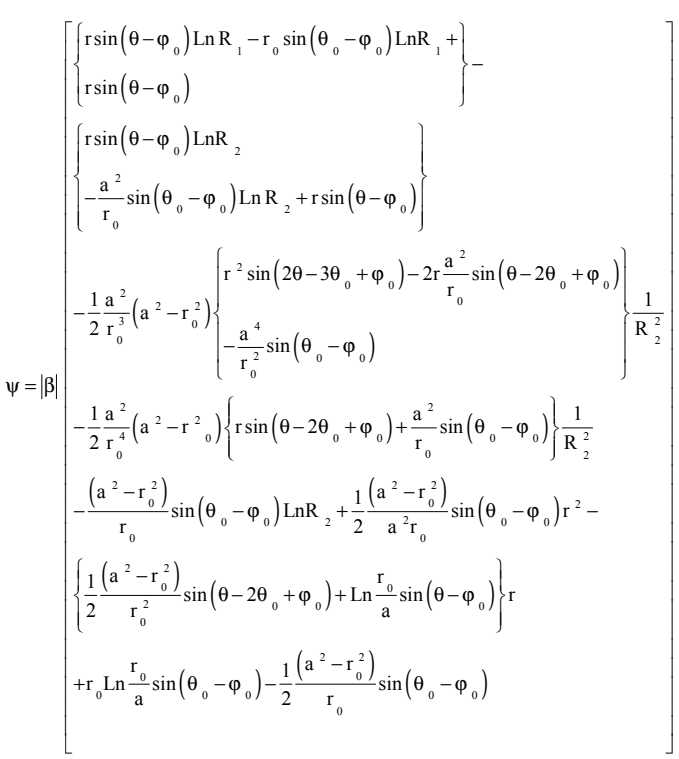


where the last two constant terms are added to make $\psi$ vanish on $\mathrm{r}=\mathrm{a}$, and where $\mathrm{r}=|\mathrm{z}|, \mathrm{R}_{1}=\left|\mathrm{z}-\mathrm{z}_{0}\right|, \mathrm{R}_{2}=\left|\mathrm{z}-\frac{\mathrm{a}}{\overline{\mathrm{z}}_{0}}\right|, \quad \mathrm{r}_{0}=\left|\mathrm{z}_{0}\right|, \theta=\arg \mathrm{z}, \theta_{0}=\arg \mathrm{z}_{0}$ and $\varphi_{0}=\arg \beta$.

A relative concise form of the stream function (31) may be given by

$$
\psi=|\beta|\left[\begin{array}{c}
r\left(\operatorname{LnR} R_{1}-\operatorname{Ln} \frac{r_{0}}{a^{2}} R_{2}\right) \sin \left(\theta-\theta_{0}\right)-\left(\begin{array}{c}
r_{0}\left(\operatorname{LnR} R_{1}-\operatorname{Ln} \frac{r_{0}}{a} R_{2}\right)+ \\
\frac{1}{2} \frac{\left(a^{2}-r_{0}^{2}\right)}{r_{0}}\left(\frac{a^{2}\left(r^{2}-a^{2}\right)}{r^{2} R_{0}^{2}}-\frac{r^{2}}{a^{2}}+1\right)
\end{array}\right) \sin \left(\theta_{0}-\varphi_{0}\right) \\
-\frac{1}{2} \frac{\left(a^{2}-r_{0}^{2}\right)}{r^{2}} \frac{\left(r^{2}-a^{2}\right) r}{R_{2}^{2}} \sin \left(\theta-2 \theta_{0}+\varphi_{0}\right) .
\end{array}\right] .
$$

And thus one may be interested in verifying that the stream function (2.34) satisfies no slip conditions, $\psi=0=\frac{\partial \psi}{\partial \mathrm{r}}$ on the boundary $r=a$.

Theorem 3. Let there be a two-dimensional flow in an unbounded incompressible viscous fluid in the $\mathrm{z}-$ plane. Let the flow be characterized by the complex velocity $v_{0}(\mathrm{z}, \overline{\mathrm{z}})=\overline{\mathrm{W}}_{0}(\overline{\mathrm{z}})-\overline{\mathrm{zW}}_{0}^{\prime}(\mathrm{z})-\omega_{0}(\mathrm{z})$, whose singularities are all at a distance less than $a$ from the origin; and let $\mathrm{W}_{0}(\mathrm{z}) \sim \mathrm{o}\left(\frac{1}{\mathrm{z}}\right)$ and $\mathrm{w}_{0}(\mathrm{z}) \sim \mathrm{o}\left(\frac{1}{\mathrm{z}^{2}}\right)$ for large $|\mathrm{z}|$. Now if a circular cylinder $|z|=a$ is introduced into the flow, then the complex velocity and the stream function for the flow internal to the cylinder becomes respectively

$$
v(z, \bar{z})=v_{0}(z, \bar{z})+v_{0}^{*}(z, \bar{z})
$$

and

$$
\psi=\frac{1}{2} \mathrm{i}\left(\mathrm{z} \overline{\mathrm{W}}(\overline{\mathrm{z}})-\overline{\mathrm{zW}}(\mathrm{z})+\int \bar{\omega}(\overline{\mathrm{z}}) \mathrm{d} \overline{\mathrm{z}}-\int \omega(\mathrm{z}) \mathrm{dz}\right),
$$

where

$$
\begin{aligned}
v_{0}^{*}(z, \bar{z})= & -\frac{\bar{z}^{3}}{\mathrm{a}^{2}} \frac{\mathrm{d}}{\mathrm{d} \overline{\mathrm{z}}} \mathrm{W}_{0}\left(\frac{\mathrm{a}^{2}}{\overline{\mathrm{z}}}\right)-\overline{\mathrm{W}}_{0}\left(\frac{\mathrm{a}^{2}}{\mathrm{z}}\right)+\omega_{0}\left(\frac{\mathrm{a}^{2}}{\overline{\mathrm{z}}}\right)+ \\
& \left(\overline{\mathrm{z}}-\frac{\mathrm{a}^{2}}{\mathrm{z}}\right)\left\{\frac{1}{\mathrm{a}^{2}} \frac{\mathrm{d}}{\mathrm{dz}}\left(\mathrm{z}^{3} \frac{\mathrm{d}}{\mathrm{dz}} \overline{\mathrm{W}}_{0}\left(\frac{\mathrm{a}^{2}}{\mathrm{z}}\right)\right)-\frac{\mathrm{d}}{\mathrm{dz}} \bar{\omega}_{0}\left(\frac{\mathrm{a}^{2}}{\mathrm{z}}\right)\right\},
\end{aligned}
$$

and where

$$
\mathrm{W}(\mathrm{z})=\mathrm{W}_{0}(\mathrm{z})-\frac{\mathrm{z}^{3}}{\mathrm{a}^{2}} \frac{\mathrm{d}}{\mathrm{d} \mathrm{z}} \overline{\mathrm{W}}_{0}\left(\frac{\mathrm{a}^{2}}{\mathrm{z}}\right)+\bar{\omega}_{0}\left(\frac{\mathrm{a}^{2}}{\mathrm{z}}\right),
$$

and

$$
\omega(\mathrm{z})=\omega_{0}(\mathrm{z})+\overline{\mathrm{W}}_{0}\left(\frac{\mathrm{a}^{2}}{\mathrm{z}}\right)+\mathrm{z}^{2} \frac{\mathrm{d}^{2}}{\mathrm{dz} \mathrm{z}^{2}} \overline{\mathrm{W}}_{0}\left(\frac{\mathrm{a}^{2}}{\mathrm{z}}\right)+3 \mathrm{z} \frac{\mathrm{d}}{\mathrm{dz}} \overline{\mathrm{W}}\left(\frac{\mathrm{a}^{2}}{\mathrm{z}}\right)-\frac{\mathrm{a}^{2}}{\mathrm{z}} \frac{\mathrm{d}}{\mathrm{dz}} \bar{\omega}_{0}\left(\frac{\mathrm{a}^{2}}{\mathrm{z}}\right) .
$$

Proof: "Account into (1) the value of the complex functions $W(z)$ and $\omega(z)$ by the expressions (38) and (39) respectively, one gets the function (35) which is clearly a particular complex velocity." Next, making use of the transformation $\mathrm{z}=\frac{\mathrm{a}^{2}}{\overline{\mathrm{z}}}$ in the complex velocity (35) yields $v(z, \bar{z})=0$, on $|z|=a$; and thus the no-slip condition is satisfied. Since, by hypothesis, $\mathrm{W}_{0}(\mathrm{z}) \sim \mathrm{o}\left(\frac{1}{\mathrm{z}}\right)$ and $\omega_{0}(\mathrm{z}) \sim \mathrm{o}\left(\frac{1}{\mathrm{z}^{2}}\right)$ for large $|\mathrm{z}|$, $\mathrm{W}_{0}(\mathrm{z})$ and $\omega_{0}(\mathrm{z})$ may be expanded in Laurent's series as

$$
\begin{gathered}
\mathrm{w}_{0}(\mathrm{z})=\frac{\mathrm{b}_{1}}{\mathrm{z}^{2}}+\frac{\mathrm{b}_{2}}{\mathrm{z}^{3}}+\frac{\mathrm{b}_{3}}{\mathrm{z}^{4}}+\ldots \ldots . ., \\
\mathrm{W}_{0}(\mathrm{z})=\frac{\mathrm{a}_{1}}{\mathrm{z}}+\frac{\mathrm{a}_{2}}{\mathrm{z}^{2}}+\frac{\mathrm{a}_{3}}{\mathrm{z}^{3}}+\ldots \ldots . .
\end{gathered}
$$

where $\mathrm{a}_{1}, \mathrm{a}_{2}, \mathrm{a}_{3}$ and $b_{1}, b_{2}, b_{3} \ldots . .$, are complex constants. Substituting these series in (2.37) results in the perturbation complex velocity within the region $|z| \leq a$, as

$$
\begin{aligned}
& v_{0}^{*}(\mathrm{z}, \overline{\mathrm{z}})=2 \overline{\mathrm{b}}_{1} \frac{1}{\mathrm{a}^{2}}+\frac{1}{\mathrm{a}^{2}}\left(2 \mathrm{a}_{1}+3 \overline{\mathrm{b}}_{2} \frac{1}{\mathrm{a}^{2}}\right) \mathrm{z} \\
& +\frac{1}{\mathrm{a}^{4}}\left(7 \overline{\mathrm{a}}_{2}+4 \overline{\mathrm{b}}_{3} \frac{1}{\mathrm{a}^{2}}\right) \mathrm{z}^{2}+\mathrm{b}_{1} \frac{1}{\mathrm{a}^{4}} \overline{\mathrm{z}}^{2}-2 \overline{\mathrm{b}} \frac{1}{1} \frac{1}{\mathrm{a}^{4}} \mathrm{z} \overline{\mathrm{z}}+\ldots . .
\end{aligned}
$$

which implies that $v_{0}^{*}(z, \bar{z})$ has no singularities inside the region $|z| \leq a$, and has in particular, finite velocity at the origin; and all conditions being satisfied the theorem is therefore proved.

Example. A stresslet interior to a circular cylinder

Let the primary flow be due to a stresslet of strength $\alpha$ at the point $\mathrm{z}_{0}$, with $\left|\mathrm{z}_{0}\right|<\mathrm{a}$, in an incompressible viscous fluid; the complex velocity [11] generated by this singularity in the fluid is given by

$$
v_{0}(\mathrm{z}, \overline{\mathrm{z}})=\frac{1}{2}\left(\bar{\alpha} \frac{1}{\overline{\mathrm{z}}-\overline{\mathrm{z}}_{0}}+\alpha \frac{\overline{\mathrm{z}}-\overline{\mathrm{z}}_{0}}{\left(\mathrm{z}-\mathrm{z}_{0}\right)^{2}}\right),
$$

which may be written in the form

$$
v_{0}(z, \bar{z})=\bar{W}_{0}(\bar{z})-\bar{z} W_{0}^{\prime}(z)-\omega_{0}(z)
$$

where

$$
\mathrm{W}_{0}(\mathrm{z})=\frac{1}{2} \alpha \frac{1}{\mathrm{z}-\mathrm{z}_{0}}
$$

and

$$
\omega_{0}(\mathrm{z})=\frac{1}{2} \alpha \overline{\mathrm{z}}_{0} \frac{1}{\left(\mathrm{z}-\mathrm{z}_{0}\right)^{2}} .
$$

A direct calculation shows that 
$\mathrm{W}_{0}(\mathrm{z}) \sim \mathrm{o}\left(\frac{1}{\mathrm{z}}\right)$ and $_{0}(\mathrm{z}) \sim \mathrm{o}\left(\frac{1}{\mathrm{z}^{2}}\right)$ for large $|\mathrm{z}|$, Thus the theorem 3 applies here. Therefore, after the insertion of the cylinder $|z|=a$ into the basic flow represented by (2.43), the complex velocity for the flow inside the cylinder becomes

$$
" v(\mathrm{z}, \overline{\mathrm{z}})=v_{0}(\mathrm{z}, \overline{\mathrm{z}})+v_{0}^{*}(\mathrm{z}, \overline{\mathrm{z}})
$$

Where

$$
\begin{aligned}
& v_{0}^{*}(\mathrm{z}, \overline{\mathrm{z}})=\left(\frac{1}{2} \bar{\alpha} \frac{1}{\bar{z}_{0}}+\frac{1}{2} \alpha \frac{z_{0}}{\mathrm{z}_{0}^{2}}-\alpha \frac{a^{2}}{\mathrm{z}_{0}^{3}}\right)-\frac{1}{2} \alpha \frac{1}{\mathrm{z}_{0}^{2}} \bar{z}- \\
& \frac{1}{2} \alpha \frac{a^{2}}{\mathrm{z}_{0}^{3}}\left(\frac{3 a^{2}}{z_{0}}-2 \bar{z}_{0}\right) \frac{1}{\left(\bar{z}-\frac{a^{2}}{\bar{z}_{0}}\right)}+\frac{1}{2} \alpha \frac{a^{4}}{z_{0}^{4}}\left(\bar{z}_{0}-\frac{a^{2}}{z_{0}}\right) \frac{1}{\left(\bar{z}-\frac{a^{2}}{z_{0}}\right)^{2}}+\frac{1}{2} \bar{\alpha} \frac{a^{2}}{\bar{z}_{0}^{2}} \frac{1}{\left(z-\frac{a^{2}}{\bar{z}_{0}}\right)} \\
& +\frac{1}{2} \bar{\alpha} \frac{1}{\bar{z}_{0}^{2}} \bar{z}\left\{1-\frac{a^{2}}{\bar{z}_{0}}\left(\frac{3 a^{2}}{\bar{z}_{0}}-2 z_{0}\right) \frac{1}{\left(z-\frac{a^{2}}{\bar{z}_{0}}\right)^{2}}+\frac{2 a^{4}}{\bar{z}_{0}^{2}}\left(z_{0}-\frac{a^{2}}{\bar{z}_{0}}\right) \frac{1}{\left(z-\frac{a^{2}}{\bar{z}_{0}}\right)^{3}}\right\} \\
& -\frac{1}{2} \bar{\alpha} \frac{a^{2}}{\bar{z}_{0}^{2}}\left\{\frac{1}{z-\frac{a^{2}}{\bar{z}_{0}}-\frac{a^{2}}{\bar{z}_{0}}} \frac{1}{\left(z-\frac{a^{2}}{\bar{z}_{0}}\right)^{2}}-\frac{2 a^{2}}{\bar{z}_{0}}\left(\frac{a^{2}}{\bar{z}_{0}}-z_{0}\right) \frac{1}{\left(z-\frac{a^{2}}{\bar{z}_{0}}\right)^{3}}\right\},
\end{aligned}
$$

which upon the appropriate reduction can be given a standard form

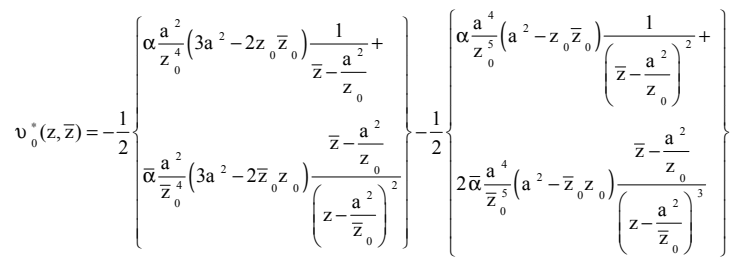

$$
\begin{aligned}
& -\frac{3}{2} \bar{\alpha} \frac{a^{4}}{z_{0} \bar{z}_{0}^{4}}\left(a^{2}-\bar{z}_{0} z_{0}\right) \frac{1}{\left(z-\frac{a^{2}}{\bar{z}_{0}}\right)^{2}}-\bar{\alpha} \frac{a^{4}}{z_{0} \bar{z}_{0}^{5}}\left(a^{2}-\bar{z}_{0} z_{0}\right)^{2} \frac{1}{\left(z-\frac{a^{2}}{\bar{z}_{0}}\right)^{3}} \\
& +\frac{1}{2} \frac{1}{z_{0}^{2} \bar{z}_{0}^{2}}\left(\bar{\alpha} \bar{z}_{0}^{2}-\alpha \bar{z}_{0}^{2}\right) \bar{z}+\left(\frac{1}{2} \bar{\alpha} \frac{1}{\bar{z}_{0}}+\frac{1}{2} \alpha \frac{\bar{z}}{z_{0}^{2}}-\alpha \frac{a^{2}}{z_{0}^{3}}\right) .
\end{aligned}
$$

The terms in (49) give the image system outside the boundary $|z|=a$, which is consisted of (i) - (v):

(1) a stresslet of strength, $-\bar{\alpha} \frac{a^{2}}{\bar{z}_{0}^{4}}\left(3 a^{2}-2 z_{0} \bar{z}_{0}\right)$ at the point $\frac{\mathrm{a}^{2}}{\overline{\mathrm{z}}_{0}}$,

(2) a stressing quadrupole of strength $-2 \bar{\alpha} \frac{\mathrm{a}^{4}}{\overline{\mathrm{z}}_{0}^{5}}\left(\mathrm{a}^{2}-\overline{\mathrm{z}}_{0} \mathrm{z}_{0}\right)$ at the point $\frac{\mathrm{a}^{2}}{\overline{\mathrm{z}}_{0}}$,

(3) a potential doublet of strength, $-\frac{3}{2} \bar{\alpha} \frac{\mathrm{a}^{4}}{\mathrm{z}_{0} \overline{\mathrm{z}}_{0}^{4}}\left(\mathrm{a}^{2}-\overline{\mathrm{z}}_{0} \mathrm{z}_{0}\right)$ at the point $\frac{\mathrm{a}^{2}}{\overline{\mathrm{z}}_{0}}$, a potential quadrupole of strength, $-\bar{\alpha} \frac{\mathrm{a}^{4}}{\mathrm{z}_{0} \overline{\mathrm{Z}}_{0}^{5}}\left(\mathrm{a}^{2}-\mathrm{z}_{0} \overline{\mathrm{Z}}_{0}\right)^{2}$ at the same point,
(4) a shear flow, $\frac{1}{2} \frac{1}{z_{0}^{2} \bar{z}_{0}^{2}}\left(\bar{\alpha} z_{0}^{2}-\alpha \bar{z}_{0}^{2}\right) \bar{z}$ and

(5) a uniform stream $\left(\frac{1}{2} \bar{\alpha} \frac{1}{\bar{z}_{0}}+\frac{1}{2} \alpha \frac{\bar{z}_{0}}{\mathrm{z}_{0}^{2}}-\alpha \frac{\mathrm{a}^{2}}{\mathrm{z}_{0}^{3}}\right)$.

The stream function corresponding to the complex velocity (47) can found by the similar way of having the expression (33) and this is

$$
\begin{aligned}
& \psi=-|\alpha| \frac{1}{2}\left\{\mathrm{r}^{2} \sin \left(2 \theta-\varphi_{0}\right)-2 \mathrm{rr}_{0} \sin \left(\theta+\theta_{0}-\varphi_{0}\right)+\mathrm{r}_{0}^{2} \sin \left(2 \theta_{0}-\varphi_{0}\right)\right\} \frac{1}{\mathrm{R}_{1}^{2}} \\
& +|\alpha| \frac{1}{2} \frac{a^{2}}{r_{0}^{4}}\left(3 a^{2}-2 r_{0}^{2}\right)\left\{\begin{array}{l}
r^{2} \sin \left(2 \theta-4 \theta_{0}+\varphi_{0}\right) \\
-2 r \frac{a^{2}}{r_{0}} \sin \left(\theta-3 \theta_{0}+\varphi_{0}\right)+\frac{a^{4}}{r_{0}^{2}} \sin \left(-2 \theta_{0}+\varphi_{0}\right)
\end{array}\right\} \frac{1}{R_{2}^{2}} \\
& +|\alpha| \frac{1}{2} \frac{\mathrm{a}^{4}}{\mathrm{r}_{0}^{5}}\left(\mathrm{a}^{2}-\mathrm{r}_{0}^{2}\right)\left\{\begin{array}{l}
\mathrm{r}^{3} \sin \left(3 \theta-5 \theta_{0}+\varphi_{0}\right)-3 \mathrm{a}^{2} \mathrm{r}^{2} \frac{1}{\mathrm{r}_{0}} \sin \left(2 \theta-4 \theta_{0}+\varphi_{0}\right) \\
+3 \mathrm{r} \frac{\mathrm{a}^{4}}{\mathrm{r}_{0}^{2}} \sin \left(\theta-3 \theta_{0}+\varphi_{0}\right)-\frac{\mathrm{a}^{6}}{\mathrm{r}_{0}^{3}} \sin \left(-2 \theta_{0}+\varphi_{0}\right)
\end{array}\right\} \frac{1}{\mathrm{R}_{2}^{4}} \\
& +|\alpha| \frac{3}{2} \frac{\mathrm{a}^{4}}{\mathrm{r}_{0}^{3}}\left(\mathrm{a}^{2}-\mathrm{r}_{0}^{2}\right)\left\{\mathrm{r} \sin \left(\theta-3 \theta_{0}+\varphi_{0}\right)-\frac{\mathrm{a}^{2}}{\mathrm{r}_{0}} \sin \left(-2 \theta_{0}+\varphi_{0}\right)\right\} \frac{1}{\mathrm{R}_{2}^{2}} \\
& +|\alpha| \frac{1}{2} \frac{\mathrm{a}^{4}}{\mathrm{r}_{0}^{6}}\left(\mathrm{a}^{2}-\mathrm{r}_{0}^{2}\right)^{2}\left\{\begin{array}{l}
\mathrm{r}^{2} \sin \left(2 \theta-4 \theta_{0}+\varphi_{0}\right) \\
-2 \mathrm{r} \frac{\mathrm{a}^{2}}{\mathrm{r}_{0}} \sin \left(\theta-3 \theta_{0}+\varphi_{0}\right)+\frac{\mathrm{a}^{4}}{\mathrm{r}_{0}^{2}} \sin \left(-2 \theta_{0}+\varphi_{0}\right)
\end{array}\right\} \frac{1}{\mathrm{R}_{2}^{4}} \\
& -|\alpha| \frac{1}{2 \mathrm{r}_{0}^{2}} \mathrm{r}^{2} \sin \left(2 \theta_{0}-\varphi_{0}\right) \\
& +|\alpha| \frac{1}{2 \mathrm{r}_{0}^{3}} \mathrm{r}\left\{\left(\mathrm{r}_{0}^{2}+2 \mathrm{a}^{2}\right) \sin \left(\theta-3 \theta_{0}+\varphi_{0}\right)-\mathrm{r}_{0}^{2} \sin \left(\theta+\theta_{0}-\varphi_{0}\right)\right\} \\
& +|\alpha| \frac{1}{2 \mathrm{r}_{0}^{2}} \mathrm{a}^{2} \sin \left(2 \theta_{0}-\varphi_{0}\right),
\end{aligned}
$$

where

$$
\begin{aligned}
& r=|z|, R_{1}=\left|z-z_{0}\right|, R_{2}=\left|z-\frac{a^{2}}{\bar{z}_{0}}\right|, r_{0}=\left|z_{0}\right|, \theta=\arg z, \\
& \theta_{0}=\arg z_{0}, \text { and } \varphi_{0}=\arg \alpha .
\end{aligned}
$$

\section{Conclusion}

Except in a few simple cases the solution of fluid mechanical problems involving an arbitrary boundary is still, in general exceedingly difficult. In the case of twodimensional slow flow theory there is a complex variable circle theorem [1] for the solutions of Stokes flows due to singularities outside a circular cylinder which corresponds to Milne-Thomson's circle theorem [2, 3] for potential flow outside the same cylinder, in the inviscid flow theory. Again it is notable that the same complex variable circle theorem can solve, in particular, some particular Stokes flow problems which cannot be done by the application of the real variable circle theorem $[4,5]$. Moreover, the "condition for zero perturbation velocity" referred to in the former theorem may suggest, in many cases, relatively easily the strengths or positions or both, of the singularities of the basic flow so that the viscous flows outside the circular boundary exist. Besides these type of solutions, many relevant solutions regarding 
this topic are also provided in this paper. Here our object is to study Stokes flow interior to a circular cylinder in the light of the complex variable theory and to establish a number of complex variable circle theorems for slow viscous fluid motion within a circular cylinder in terms of the complex velocities of the fundamental singularities found in Chowdhury and Sen [11]; these theorems also correspond to the complex variable circle theorems for potential flow [12, $13]$ in an inviscid fluid within the same cylinder.

\section{Appendix}

(1) Calculation of the stream functions of the singularities constituting the complex velocity (14)

Let $\psi_{1}, \psi_{2}, \psi_{3}, \psi_{4}, \psi_{5}$ and $\psi_{6}$ be respectively the stream functions of the basic rotlet, the image rotlet, stresslet, potential doublet, shear flow and the uniform stream, referred to (14).

By taking $\mathrm{W}(\mathrm{z})=0$ and $\omega(\mathrm{z})=\omega_{0}(\mathrm{z})$ in the stream function (4),

$$
\psi_{1}=\frac{1}{2} \mathrm{i}\left(\int \bar{\omega}_{0}(\bar{z}) \mathrm{d} \bar{z}-\int \omega_{0}(\mathrm{z}) \mathrm{dz}\right), \omega_{0}(\mathrm{z})=-\frac{\mathrm{ik}}{\mathrm{z}-\mathrm{z}_{0}} .
$$

i. e.,

$$
\psi_{1}=-\mathrm{kInR}_{1}, \text { where } \mathrm{R}_{1}=\left|\mathrm{z}-\mathrm{z}_{0}\right|
$$

Similarly,

$$
\psi_{2}=\mathrm{kInR}_{2}, \text { where } \mathrm{R}_{2}=\left|\mathrm{z}-\frac{\mathrm{a}^{2}}{\overline{\mathrm{z}}_{0}}\right| .
$$

Next for the determination of $\psi_{3}$, the complex velocity of the stresslet, shown in (14) is expressed as

$$
i k\left\{\frac{a^{2}}{z_{0}^{2}} \frac{1}{\bar{z}-\frac{a^{2}}{z_{0}}}-\frac{a^{2}}{\bar{z}_{0}^{2}} \frac{\bar{z}-\frac{a^{2}}{z_{0}}}{\left(z-\frac{a^{2}}{\bar{z}_{0}}\right)^{2}}\right\}=\bar{W}(\bar{z})-\bar{z} W^{\prime}(z)-\omega(z)
$$

where

$$
W(z)=-i k \frac{a^{2}}{\bar{z}_{0}^{2}} \frac{1}{z-\frac{a^{2}}{\bar{z}_{0}}} \text { and } \omega(z)=-i k \frac{a^{2}}{\bar{z}_{0}^{2}} \frac{a^{2}}{z_{0}} \frac{1}{\left(z-\frac{a^{2}}{\bar{z}_{0}}\right)^{2}} .
$$

Thus using these expressions in (4) yields

$$
\psi_{3}=-\mathrm{k} \frac{\mathrm{a}^{2}}{\mathrm{r}_{0}^{2}}\left\{\mathrm{r}^{2} \cos 2\left(\theta-\theta_{0}\right)-2 \mathrm{r} \frac{\mathrm{a}^{2}}{\mathrm{r}_{0}} \cos \left(\theta-\theta_{0}\right)+\frac{\mathrm{a}^{4}}{\mathrm{r}_{0}^{2}}\right\} \frac{1}{\mathrm{R}_{2}^{2}},
$$

Where $\mathrm{r}_{0}=\left|\mathrm{z}_{0}\right|$, and $\theta_{0}=\arg \mathrm{z}_{0}$.

Then the stream function

$$
\psi_{4}=\frac{1}{2} \mathrm{i}\left(\int \bar{\omega}_{0}(\bar{z}) \mathrm{d} \bar{z}-\int \omega_{0}(\mathrm{z}) \mathrm{dz}\right)
$$

where

$$
\omega(\mathrm{z})=-\mathrm{ik} \frac{\mathrm{a}^{2}}{\overline{\mathrm{z}}_{0}}\left(1-\frac{\mathrm{a}^{2}}{\mathrm{z}_{0} \overline{\mathrm{z}}_{0}}\right) \frac{1}{\left(\mathrm{z}-\frac{\mathrm{a}^{2}}{\overline{\mathrm{z}}_{0}}\right)^{2}} .
$$

The evaluation of the stream function (57) is straightforward, giving

$$
\psi_{4}=-\frac{\mathrm{ka}^{2}}{\mathrm{r}_{0}^{3}}\left(\mathrm{a}^{2}-\mathrm{r}_{0}^{2}\right)\left\{\mathrm{r} \cos \left(\theta-\theta_{0}\right)-\frac{\mathrm{a}^{2}}{\mathrm{r}_{0}}\right\} \frac{1}{\mathrm{R}_{2}^{2}} .
$$

In order to determine the stream function $\psi_{5}$, of the shear flow we express its complex velocity as

$$
\frac{\mathrm{ik}}{\mathrm{a}^{2}} \overline{\mathrm{z}}=\overline{\mathrm{W}}(\overline{\mathrm{z}})-\overline{\mathrm{zW}}(\mathrm{z})
$$

Where $W(z)=-\frac{i k}{2 a^{2}} z$. So the same stream function is given by the formula (4) with $\omega(\mathrm{z})=0$; and thus

$$
\psi_{5}=\frac{1}{2} \mathrm{i}(\mathrm{z} \overline{\mathrm{W}}(\overline{\mathrm{z}})-\overline{\mathrm{zW}}(\mathrm{z}))=-\frac{1}{2} \frac{\mathrm{k}}{\mathrm{a}^{2}} \mathrm{r}^{2}
$$

Similarly, the stream function $\psi_{6}$, of the uniform stream is obtained by the formula

$$
\psi_{6}=\frac{1}{2} \mathrm{i}\left(\int \bar{\omega}(\bar{z}) \mathrm{d} \bar{z}-\int \omega(\mathrm{z}) \mathrm{d} \mathrm{z}\right)
$$

in which $\omega(\mathrm{z})=-\frac{\mathrm{ik}}{\mathrm{z}_{0}}$; and this clearly gives

$$
\psi_{6}=-\frac{\mathrm{k}}{\mathrm{r}_{0}} \mathrm{r} \cos \left(\theta-\theta_{0}\right)
$$

(2) Calculation of the stream functions of the singularities constituting the complex velocity (32)

Let $\psi_{7}, \psi_{8}, \psi_{9}, \psi_{10}, \psi_{11}, \psi_{12}$, and $\psi_{13}$ be the stream functions of the basic stokeslet, the image stokeslet, stresslet, potential doublet, rotlet, shear flow and uniform stream, all of which make the complex velocity (32). The expression (27) is the complex velocity of the basic stokeslet. Thus by the formula (18) the corresponding stream function is

$$
\psi_{7}=\frac{1}{2} \mathrm{i}\left(\mathrm{z} \overline{\mathrm{W}}_{0}(\overline{\mathrm{z}})-\overline{\mathrm{z}} \mathrm{W}_{0}(\mathrm{z})+\int \bar{\omega}_{0}(\overline{\mathrm{z}}) \mathrm{d} \overline{\mathrm{z}}-\int \omega_{0}(\mathrm{z}) \mathrm{d} \mathrm{z}\right)
$$

where $\mathrm{W}_{0}(\mathrm{z})$ and $\omega_{0}(\mathrm{z})$ stand for the functions (29) and (30). Evaluating the integrals in (64) in a straightforward manner yields the stream function for the basic Stokeslet, that 
is

$$
\psi_{7}=|\beta|\left\{\mathrm{r} \sin \left(\theta-\varphi_{0}\right) \mathrm{LnR}{ }_{1}-\mathrm{r}_{0} \sin \left(\theta_{0}-\varphi_{0}\right) \mathrm{LnR}{ }_{1}+\mathrm{r} \sin \left(\theta-\varphi_{0}\right)\right\},
$$

where $\varphi_{0}=\arg \beta$. Similarly, the stream function for the image stokeslet is

$$
\psi_{8}=-|\beta|\left\{r \sin \left(\theta-\varphi_{0}\right) \operatorname{LnR}{ }_{2}-r_{0} \sin \left(\theta_{0}-\varphi_{0}\right) L_{2 n R}+r \sin \left(\theta-\varphi_{0}\right)\right\} .
$$

The complex velocity of the stresslet can be expressed as

$$
\begin{gathered}
\frac{1}{2} \beta \frac{\mathrm{a}^{2}}{\mathrm{z}_{0}^{2}}\left(\frac{\mathrm{a}^{2}}{\mathrm{z}_{0}}-\overline{\mathrm{z}}_{0}\right) \frac{1}{\overline{\mathrm{z}}-\frac{\mathrm{a}^{2}}{\mathrm{z}_{0}}}+\frac{1}{2} \bar{\beta} \frac{\mathrm{a}^{2}}{\overline{\mathrm{z}}_{0}^{2}}\left(\frac{\mathrm{a}^{2}}{\overline{\mathrm{z}}_{0}}-\mathrm{z}_{0}\right)\left(\overline{\mathrm{z}}-\frac{\mathrm{a}^{2}}{\mathrm{z}_{0}}\right) \frac{1}{\left(\mathrm{z}-\frac{\mathrm{a}^{2}}{\overline{\mathrm{z}}_{0}}\right)^{2}} \\
=\bar{W}(\bar{z})-\bar{z} W^{\prime}(\mathrm{z})-\omega(\mathrm{z}),
\end{gathered}
$$

where

$$
\mathrm{W}(\mathrm{z})=\frac{1}{2} \bar{\beta} \frac{\mathrm{a}^{2}}{\overline{\mathrm{z}}_{0}^{2}}\left(\frac{\mathrm{a}^{2}}{\overline{\mathrm{z}}_{0}}-\mathrm{z}_{0}\right) \frac{1}{\left.\mathrm{z}-\frac{\mathrm{a}^{2}}{\overline{\mathrm{z}}_{0}}\right)}
$$

and

$$
\omega(\mathrm{z})=\frac{1}{2} \bar{\beta} \frac{\mathrm{a}^{2}}{\overline{\mathrm{z}}_{0}^{2}}\left(\frac{\mathrm{a}^{2}}{\overline{\mathrm{z}}_{0}}-\mathrm{z}_{0}\right)\left(\frac{\mathrm{a}^{2}}{\mathrm{z}_{0}}\right) \frac{1}{\left.\mathrm{z}-\frac{\mathrm{a}^{2}}{\overline{\mathrm{z}}_{0}}\right)^{2}} .
$$

Making use of the last results in the formula (18) leads to the stream function

$$
\psi_{9}=-\frac{1}{2}|\beta| \frac{a^{2}}{r_{0}^{3}}\left(a^{2}-r_{0}^{2}\right)\left\{\begin{array}{l}
r^{2} \sin \left(2 \theta-3 \theta_{0}+\varphi_{0}\right)-2 \frac{a^{2}}{r_{0}} \sin \left(\theta-2 \theta_{0}+\varphi_{0}\right) \\
-\frac{a^{4}}{r_{0}^{2}} \sin \left(\theta_{0}-\varphi_{0}\right)
\end{array}\right\} \frac{1}{R_{2}^{2} .}
$$

The stream function for the potential doublet is given by

$$
\psi_{10}=\frac{1}{2} \mathrm{i}\left(\int \bar{\omega}(\bar{z}) \mathrm{d} \overline{\mathrm{z}}-\int \omega(\mathrm{z}) \mathrm{d} \mathrm{z}\right)
$$

where

$$
\omega(\mathrm{z})=-\frac{1}{2} \beta \frac{\mathrm{a}^{2}}{\mathrm{z}_{0} \overline{\mathrm{z}}^{3}}\left(\mathrm{a}^{2}-\mathrm{z} \overline{\mathrm{z}}_{0}\right)^{2} \frac{1}{\left(\mathrm{z}-\frac{\mathrm{a}^{2}}{\overline{\mathrm{z}}_{0}}\right)^{2}} .
$$

By using (72) in (71) we get

$$
\psi_{10}=-\frac{1}{2} \frac{\mathrm{a}^{2}}{\mathrm{r}_{0}^{2}}\left(\mathrm{a}^{2}-\mathrm{r}_{0}^{2}\right)^{2}\left\{\mathrm{r} \sin \left(\theta-2 \theta_{0}+\varphi_{0}\right)+\frac{\mathrm{a}^{2}}{\mathrm{r}_{0}} \sin \left(\theta_{0}-\varphi_{0}\right)\right\} \frac{1}{\mathrm{R}_{2}^{2}}
$$

Similarly, the stream function for the rotlet is calculated by using the formula (71) with

$$
\omega(\mathrm{z})=-\frac{1}{2}\left(\beta \overline{\mathrm{z}}_{0}-\bar{\beta} \mathrm{z}_{0}\right)\left(1-\frac{\mathrm{a}^{2}}{\mathrm{z}_{0} \overline{\mathrm{z}}_{0}}\right) \frac{1}{\left.\mathrm{z}-\frac{\mathrm{a}^{2}}{\overline{\mathrm{z}}_{0}}\right)}
$$

and it emerges as

$$
\psi_{11}=-|\beta|\left(\mathrm{a}^{2}-\mathrm{r}_{0}^{2}\right) \frac{1}{\mathrm{r}_{0}} \sin \left(\theta_{0}-\varphi_{0}\right) \mathrm{LnR}_{2} .
$$

To find out the stream function $\psi_{12}$, for the shear flow, its complex velocity is written as

$$
\frac{1}{2}\left(\beta \overline{\mathrm{z}}_{0}-\bar{\beta} \mathrm{z}_{0}\right)\left(\frac{1}{\mathrm{z}_{0} \overline{\mathrm{z}}_{0}}-\frac{1}{\mathrm{a}^{2}}\right) \overline{\mathrm{z}}=\overline{\mathrm{W}}(\overline{\mathrm{z}})-\overline{\mathrm{z}} \mathrm{W}^{\prime}(\mathrm{z}),
$$

where

$$
\mathrm{W}(\mathrm{z})=\frac{1}{4}\left(\bar{\beta} \mathrm{z}_{0}-\beta \overline{\mathrm{z}}_{0}\right)\left(\frac{1}{\mathrm{z}_{0} \overline{\mathrm{z}}_{0}}-\frac{1}{\mathrm{a}^{2}}\right) \mathrm{z},
$$

Thus, using (77) in the formula (2.18) with $\omega(\mathrm{z})=0$, yields

$$
\psi_{12}=\frac{1}{2}|\beta| \frac{\left(\mathrm{a}^{2}-\mathrm{r}_{0}^{2}\right)}{\mathrm{a}^{2} \mathrm{r}_{0}} \mathrm{r}^{2} \sin \left(\theta_{0}-\varphi_{0}\right)
$$

Finally,

substituting

$\omega(\mathrm{z})=\frac{1}{2} \beta\left(\frac{\mathrm{a}^{2}}{\mathrm{z}_{0}^{2}}-\frac{\overline{\mathrm{z}}_{0}}{\mathrm{z}_{0}}\right)+\frac{1}{2} \bar{\beta} \operatorname{Ln}\left(\frac{\mathrm{z}_{0} \overline{\mathrm{z}}_{0}}{\mathrm{a}^{2}}\right)$ and $\mathrm{W}(\mathrm{z})=0 \quad$ in (18) the stream function for the uniform stream may be given by

$$
\psi_{13}=-|\beta|\left\{\frac{\left(\mathrm{a}^{2}-\mathrm{r}_{0}^{2}\right)}{2 \mathrm{r}_{0}^{2}} \sin \left(\theta-2 \theta_{0}+\varphi_{0}\right)+\operatorname{Ln} \frac{\mathrm{r}_{0}}{\mathrm{a}} \sin \left(\theta-\varphi_{0}\right)\right\} \mathrm{r} .
$$

\section{References}

[1] Sen S K, A reflection theorem for plane Stokes flow, (under consideration for publication), presented in $15^{\text {th }}$ Mathematic conf. 29 to 31 December 2007, Dhaka, Bangladesh.

[2] L. M. Milne- Thomson, Hydrodynamical images, Proc. Camb. Phil. Soc., 36, 1940, 246-247.

[3] L. M. Milne-Thomson, Theoretical Hydrodynamics, $5^{\text {th }}$ Edition 1972, pp. 157-158., 181-190.

[4] A. Avudainayagam and B. Jothiram, A circle theorem for plane Stokes flows, Q. J. Mech. A ppl. Math., 41, 1988, pt.3, 383-393.

[5] S. K. Sen,Circle theorems for steady Stokes flows, Z. angew. Math. Phys. (ZAMP), 40, 1989, 139-146.

[6] K. B. Ranger, Eddies in two-dimensional Stokes flow, Int. J. Eng. Sci., 18, 1980. 
[7] V. V. Meleshko and H. Arof, A blinking rotlet model for chaotic advection, Phys. Fluids A, 8, 1996, 3215.

[8] Chowdhury G A H, Studies on flows in a viscous fluid, Ph. D Thesis, 2001, Research centre for Mathematical and Physical Sciences, (RCMPS), University of Chittagong, Bangladesh.

[9] Chowdhury G A H and Sen S K, A note on Stokes flow within a circular cylinder, GANIT; J. Bangladesh Math. Soc, 26, 2006, 43-50.

[10] Prabir Daripa and D. Palaniappan, Singularity induced exterior and interior Stokes flows, Phys. Fiuids 13 (11), 2001, 3134-3154.
[11] Chowdhury G A H and Sen S K, Stokes flow before a plane boundary,Indian J. pure appl. Math., 34 (2), 2003, 353-361.

[12] Sen S K, Studies on flows in viscous and non-viscous Fluids, $\mathrm{Ph}$. D. Thesis, 1993, Research Centre for Mathematical and Physical Sciences, (RCMPS), University of Chittagong, Bangladesh.

[13] F. Chorlton, Text Book of Fluid Dynamics, Van Nostrand Reinhold, London, 1967, PP187-188.

[14] W. E. Langlois, Slow Viscous Flow, The Macmillan Company, New York, 1964, p. 159. 